\title{
Improving recall in an associative neural network model of the hippocampus ${ }^{*}$
}

\author{
Nikolaos Andreakos ${ }^{1}$, Shigang Yue ${ }^{1[0000-0002-1899-6307]}$ and Vassilis Cutsuridis ${ }^{1,2, *}[0000-$ \\ 0001-9005-0260] \\ ${ }^{1}$ School of Computer Science, University of Lincoln, Lincoln, U.K. \\ ${ }^{2}$ Lincoln Sleep Research Center, University of Lincoln, Lincoln, U.K. \\ \{nandreakos, syue, vcutsuridis\}@lincoln.ac.uk \\ ${ }^{*}$ Corresponding author
}

\begin{abstract}
The mammalian hippocampus is involved in auto-association and hetero-association of declarative memories. We employed a bio-inspired neural model of hippocampal CA1 region to systematically evaluate its mean recall quality against different number of stored patterns, overlaps and active cells per pattern. Model consisted of excitatory (pyramidal cells) and four types of inhibitory cells: axo-axonic, basket, bistratified, and oriens lacunosum-moleculare cells. Cells were simplified compartmental models with complex ion channel dynamics. Cells' firing was timed to a theta oscillation paced by two distinct neuronal populations exhibiting highly regular bursting activity, one tightly coupled to the trough and the other to the peak of theta. During recall excitatory input to network excitatory cells provided context and timing information for retrieval of previously stored memory patterns. Dendritic inhibition acted as a non-specific global threshold machine that removed spurious activity during recall. Simulations showed recall quality improved when the network's memory capacity increased as the number of active cells per pattern decreased. Furthermore, increased firing rate of a presynaptic inhibitory threshold machine inhibiting a network of postsynaptic excitatory cells has a better success at removing spurious activity at the network level and improving recall quality than increased synaptic efficacy of the same threshold machine on the same network of excitatory cells, while keeping its firing rate fixed.
\end{abstract}

Keywords: Associative memories, Brain, Inhibition.

\footnotetext{
* Acknowledgements: This work was supported in part by EU Horizon 2020 through Project ULTRACEPT under Grant 778062.
} 


\section{$1 \quad$ Introduction}

Associative memory (AM) is the ability to learn and remember the relationship between items, events, places and/or objects which may be unrelated [18]. AM is one of the oldest artificial neural networks' paradigms [19-20]. In these models storing patterns was done via changes in connection strengths between artificial neurons which crudely mimicked biological ones. Old memories were recalled when a noisy, partial or complete version of a previously stored pattern was presented to the network. However, these AM devices were not very flexible. They had to be told when to store a memory pattern and when to recall it.

In 2010 a much more flexible model was introduced that controlled for itself the storage and recall of patterns of information arriving at unpredictable rates [1]. The model was based upon the many details were then known about the neuronal hippocampal circuit [21-22]. The model explored the functional roles of somatic, axonic and dendritic inhibition in the encoding and retrieval of memories in region CA1. It showed how theta modulated inhibition separated encoding and retrieval of memories in the hippocampus into two functionally independent processes. It predicted that somatic (basket cell) inhibition allowed generation of dendritic calcium spikes that promoted synaptic long-term plasticity (LTP), while minimizing cell output. Proximal dendritic (bistratified cell (BSC)) inhibition controlled both cell output and suppressed dendritic calcium spikes, thus preventing LTP, whereas distal dendritic (OLM cell) inhibition removed interference from (new and old) memory patterns trying to be encoded during recall. The mean recall quality of the model was tested as function of memory patterns stored. Recall dropped as more patterns were stored due to interference between previously stored memories. Proximal dendritic inhibition was held constant as the number of memory patterns stored was increased.

Here, we more systematically investigated the mechanisms to improve the recall performance of [1]. In particular, we examined how selective modulation of feedforward/feedback excitatory/inhibitory pathways targeting inhibitory and excitatory cells may influence the thresholding ability of dendritic inhibition to remove at the network level spurious activities, which may otherwise impair the recall performance of the network, and improve its mean recall quality as more and more overlapping memories were stored.

\section{$2 \quad$ Materials and methods}

\subsection{Neural network model}

Figure 1 depicts the simulated neural network model of region CA1 of the hippocampus. The model consisted of 100 pyramidal cells (PC), 1 axo-axonic cell (AAC), 2 basket cells (BC), 1 BSC and 1 OLM cell. The neuronal dynamics of the model cells with respect to a theta rhythm is depicted in Figure 2. Model cells were simplified compartmental models with complex ion channel dynamics. Simplified morphologies including the soma, apical and basal dendrites and a portion of the axon were used for each cell type. The biophysical properties of each cell were adapted from cell types reported in the literature, which were extensively validated against experimental data in $[4-7,13]$. 
In the model, AMPA, NMDA, GABA-A and GABA-B synapses were considered. GABA-A were present in all strata, whereas GABA-B were present in medium and distal SR and SLM dendrites. AMPA synapses were present in SLM (EC connections) and SR (CA3 connections), whereas NMDA were present only in SR (CA3 connections). The complete mathematical formalism of the model has been described elsewhere [1]. Schematic representations of model cells can be found in [12]. The dimensions of the somatic, axonic and dendritic compartments of model cells, the parameters of all passive and active ionic conductances, synaptic waveforms and synaptic conductances can be found in [12]. All simulations were performed using NEURON [8] running on a PC with eight CPUs under Windows 10.



Fig. 1 (Left) Associative neural network model of region CA1 of the hippocampus. During retrieval only PC, BSC, and OLM cells are active. AAC and BC are inactive due to strong medial septum inhibition. BSC and PC are driven on their SR dendrites by a strong CA3 excitatory input, which presented the contextual information. Red circles on PC dendrites represent active synapses, whereas black circles on PC dendrites represent inactive synapses. EC: Entorhinal cortical input; CA3: Schaffer collateral input; AAC: Axo-axonic cell; BC: basket cell; BSC: bistratified cell; OLM: oriens lacunosum-moleculare cell; SLM: stratum lacunosum moleculare; SR: stratum radiatum; SP: stratum pyramidale; SO: stratum oriens. (Right) Model CA1-PC with excitatory and inhibitory synaptic contacts on its SR dendrites.

\subsection{Inputs}

Network was driven by a CA3 Schaffer collateral excitatory input and an MS inhibitory input. The CA3 input was modelled as the firing of 20 out of 100 CA3 pyramidal cells at an average gamma frequency of $40 \mathrm{~Hz}$ (spike trains only modelled and not the explicit cells). PCs, BCs, AACs, BSCs received CA3 input in their medial SR dendrites. 
On the other hand, the MS input was modelled with the rhythmic firing of two populations of 10 septal cells each modulated at opposite phases of a theta cycle $\left(180^{\circ}\right.$ out of phase) [16]. Septal cell output was modelled as bursts of action potentials using a presynaptic spike generator. Each spike train consisted of bursts of action potentials at a mean frequency of $8 \mathrm{~Hz}$ for a half-theta cycle $(125 \mathrm{~ms})$ followed by a half-theta cycle of silence $(125 \mathrm{~ms})$. Due to $8 \%$ noise in the inter-spike intervals, the 10 spike trains in each septal population were asynchronous. $\mathrm{MS}_{2}$ input provided GABA-A inhibition to BSCs and OLMs during the encoding cycle, whereas $\mathrm{MS}_{1}$ input provided GABA-A inhibition to AACs and BCs during the retrieval cycle.


Fig. 2 Voltage traces of model cells with respect to a single theta cycle. 


\subsection{Network training and testing}

To test the recall performance of the model the methodology described in [1] was adopted. Briefly, a memory pattern was stored by generating weight matrices based on a clipped Hebbian learning rule; these weight matrices were used to pre-specify the CA3 to CA1 PC connection weights. Without loss of generality, the input (CA3) and output (CA1) patterns were assumed to be the same, with each pattern consisting of $\mathrm{N}$ $(\mathrm{N}=5$ or 10 or 20 ) randomly chosen PCs (active cells per pattern) out of the population of 100 . The 100 by 100 dimensional weight matrix was created by setting matrix entry $(i, j), w_{i j}=1$ if input $P C i$ and output $P C \mathrm{j}$ are both active in the same pattern pair; otherwise weights are 0 . Any number of pattern pairs could be stored to create this binary weight matrix. The matrix was applied to our network model by connecting a CA3 input to a CA1 PC with a high AMPA conductance $\left(\mathrm{g}_{\mathrm{AMPA}}=1.5 \mathrm{nS}\right)$ if their connection weight was 1 , or with a low conductance $\left(g_{\text {AMPA }}=0.5 \mathrm{nS}\right)$ if their connection was 0 . This approach is in line with experimental evidence that such synapses are 2state in nature [17].



Fig. 3 Set of five memory patterns with $20 \%$ overlap between them.

\subsection{Memory patterns}

We created sets of memory patterns at different sizes $(1,5,10,20)$, percent overlaps $(0 \%, 10 \%, 20 \%, 40 \%)$ and number of active cells per pattern $(5,10,20)$. A $0 \%$ overlap between 5 patterns in a set meant no overlap between patterns. A $20 \%$ overlap between 5 stored patterns meant that $0.2 * \mathrm{~N}$ cells were shared between patterns 1 and 2 , different $0.2 * \mathrm{~N}$ cells between patterns 2 and 3 , and so on (see Figure 3). For 20 active cells per 
pattern that meant that a maximum of 5 patterns could be stored by our network of 100 PCs. For 10 active cells per pattern, a maximum of 10 patterns could be stored and for 5 active cells per pattern, a maximum of 20 patterns could be stored. Similar maximum number of patterns could be stored for $10 \%, 20 \%$ and $40 \%$ overlap and 5,10 and 20 active cells per pattern, respectively. In the case of $10 \%$ overlap, 5 active cells per pattern, the maximum number of stored patterns was not an integer, so this case was excluded from our simulations.

\subsection{Recall performance measure}

The recall performance metric used for measuring the distance between the recalled output pattern, B, from the required output pattern, $\mathrm{B}^{*}$, was the correlation (i.e., degree of overlap) metric, calculated as the normalized dot product:

$$
C-\frac{B \times B^{\star}}{\left(\sum_{i-1}^{N_{B}} B_{i} \times \sum_{j^{-1}}^{N_{B}} B_{f}^{\star}\right)^{1 / 2}}
$$

where $N_{B}$ is the number of output units. The correlation takes a value between 0 (no correlation) and 1 (the vectors are identical). The higher the correlation, the better the recall performance.

\subsection{Mean recall quality}

Mean recall quality of a network model was defined as the mean value of all recall qualities estimated from each pattern presentation when an $\mathrm{N}$ number of patterns were already stored in the network. For example, when five patterns were initially stored in the network and pattern 1 is presented to the network during recall, then a recall quality value for pattern 1 was calculated. Repeating this process for each of the other patterns (pattern 2, pattern 3, pattern 4, pattern 5), a recall quality value was calculated. The mean recall quality of the network was then the mean value of these individual recall qualities.

\subsection{Model selection}

In [1], BSC inhibition to PC dendrites acted as a global non-specific threshold machine capable of removing spurious activity at the network level during recall. In [1] BSC inhibition was held constant as the number of stored patterns to PC dendrites increased. The recall quality of the model in [1] decreased as more and more memories were loaded onto the network (see Fig. 14 in [1]). To improve the recall performance of [1] we artificially modulated the synaptic strength of selective excitatory and inhibitory pathways to BSC and PC dendrites as more and more patterns were stored in the network (see Figures 1 Left and 4):

1. Model 1: Increased CA3 feedforward excitation (weight) to BSC (Fig 4A) increased the frequency of its firing rate. As a result, more IPSPs were generated in the PC dendrites producing a very strong inhibitory environment which eliminated all spurious activity. 


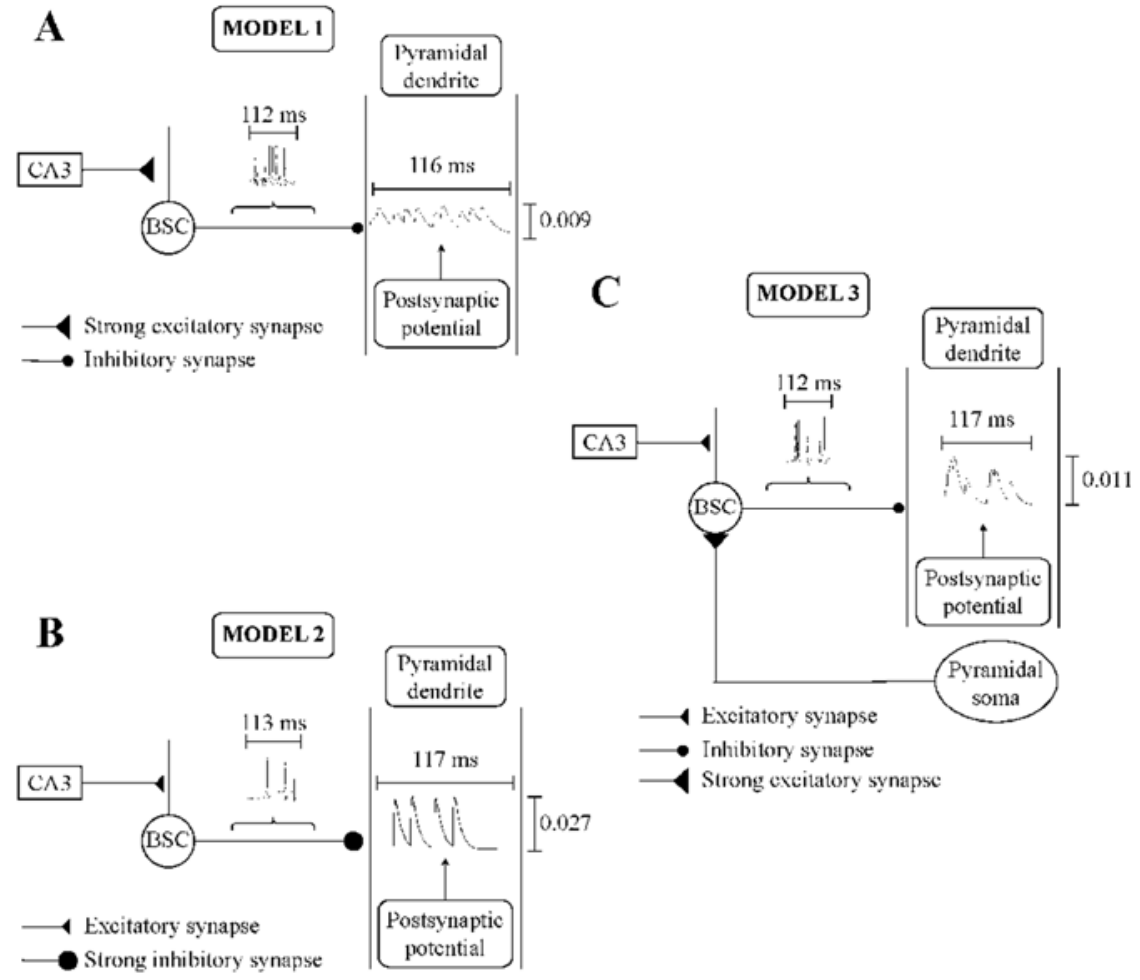

Fig. 4. Schematic drawing of presynaptic BSC firing response and inhibitory postsynaptic potentials (IPSPs) on PC dendrites in (A) 'model 1', (B) 'model 2' and (C) 'model 3'.

2. Model 2: Increased BSC feedforward inhibition (weight) to PC dendrites (Fig 4B) produced fewer IPSPs, but with greater amplitude, in the PC dendrites.

3. Model 3: Increased PC feedback excitation (weight) to BSC (Fig 4C) had a similar effect as Model 1, but with less potency.

Comparative analysis of the above three models' recall performance is depicted in Figs 5-6.

\section{Results and discussion}

A set of patterns $(1,5,10,20)$ at various percent overlaps $(0 \%, 10 \%, 20 \%, 40 \%)$ were stored by different number of 'active cells per pattern' $(5,10,20)$ without recourse to a learning rule by generating a weight matrix based on a clipped Hebbian learning rule, and using the weight matrix to prespecify the CA3 to CA1 PC connection weights. To test recall of a previously stored memory pattern in the model, the entire associated input pattern was applied as a cue in the form of spiking of active CA3 inputs (those belonging to the pattern) distributed within a gamma frequency time window. The cue 
pattern was repeated at gamma frequency $(40 \mathrm{~Hz})$. During the retrieval only the BSCs and OLM cells were switched on, whereas the AACs and BCs were switched off. The CA3 spiking drove the CA1 PCs plus the BSCs. The EC input (see Figure 1Left), which excited the apical dendrites of PCs, AACs and BCs, was disconnected during the retrieval.
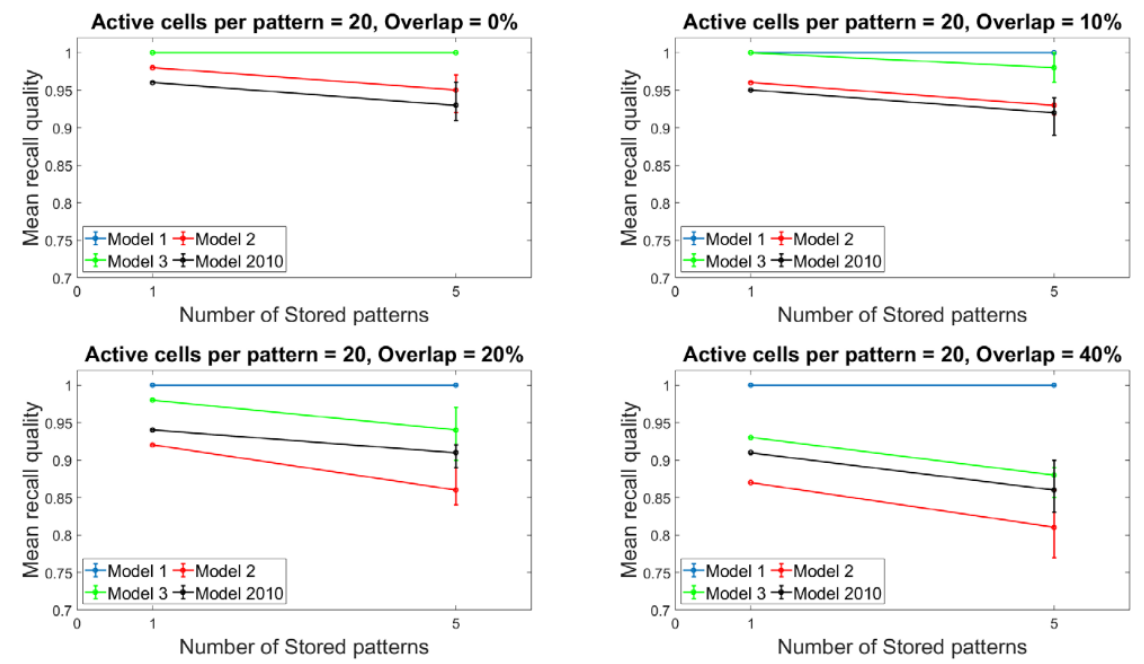

Fig. 5 Mean recall quality of 'model 1', 'model 2', 'model 3' and 'model 2010' as a function of number of stored patterns. Twenty 'active cells per pattern' in a network of 100 PCs for various percentages of overlap $(0 \%, 10 \%, 20 \%, 40 \%)$.

We can observe from Figure 5 that the recall performance of 'model 1' is best $(\mathrm{C}=$ 1) across all overlaps $(0 \%, 10 \%, 20 \%$, and $40 \%)$. Similarly, the recall performance of 'model 2' is consistently worst when compared to those of 'model 1' and 'model 3' across all overlap conditions even when only 5 patterns were stored. At $0 \%$ and $10 \%$ overlap, all three models outperformed the Cutsuridis and colleagues 2010 model [1]. At $20 \%$ and $40 \%$ overlap, model 2 did even worse than the ' 2010 model'. It is clear for large overlaps (20\% and $40 \%)$ interference effects between stored patterns cause more spurious cells to fire impairing this way the mean recall quality of the network models.

A comparison of the recall performances of models 1, 2, 3, and 2010 for different number of 'active cells per pattern' (cell assembly that code for a particular memory pattern) and number of stored patterns with $40 \%$ overlap is evident in Figure 6 . As before, 'model 1' consistently has the best performance $(\mathrm{C}=1)$ for any number of 'active cells per pattern'. As the number of 'active cells per pattern' is decreased, even at $40 \%$ overlap between stored patterns, the mean recall quality of all other three models ('Model 2', 'Model 3', 'Model 2010') increases (C 1 for all three models when number of 'active cells per pattern' is 5). This is because as the number of 'active cells per pattern' decrease, the memory capacity of the 100-by-100 dimensional weight matrix between CA3-CA1 increases (i.e. weight matrix has more " 0 's" and less "1's", so interference effects between stored patterns decrease). 
Why was 'model 1' performance so consistently better than 'model 2' and 'model 3 ' across all conditions? Why the recall quality of 'model 1 ' was always perfect $(\mathrm{C}=$ 1) even when more patterns were stored in the network, more/less 'active cells per pattern' were used to represent a memory and greater percentages of overlap between patterns were used? As we described in section "2.7 - Model selection", 'model 1' was the model where CA3 feedforward excitation to BSC was progressively increased as more and more patterns were stored, while the BSC inhibitory effect (weight) to PC dendrites was held fixed. 'Model 3' was the model where PC feedback excitation to BSC was progressively increased as more and more patterns were stored, while the BSC inhibitory effect to PC dendrites was held fixed. 'Model 2' was the model where the exact opposite took place: the inhibitory effect of BSC to PC dendrites progressively increased as more and more patterns were stored in the network, while keeping the BSC firing rate constant. In all simulations, 'model 1' outperformed 'model 3' across all conditions (overlaps and 'active pattern cells'). This was due to the fact in 'model 1' BSC was excited by $100 \mathrm{CA} 3-\mathrm{PCs}$ at high frequency $(40 \mathrm{~Hz})$, whereas in 'model 3' BSC was excited by 20 CA1-PCs that fired once or twice. Since in 'model 1' the BSC firing frequency response is higher than in 'model 3', then the postsynaptic effect of BSC on the PC dendrites in 'model 1' is higher in frequency and duration (but not in amplitude) than in 'model 3' (see Figure 4A \& 4C). Thus, 'model 1' has a better success at removing spurious activities and improving recall quality than 'model 3'. Since the BSC frequency response in 'model 2' was fixed, but its postsynaptic effect (weight) on PC dendrites increased, then the amplitude of the inhibitory postsynaptic potentials (IPSPs) on PC dendrites increased (compared to the IPSP amplitudes in models 1 and 3), but their frequency response was low (lower than in models 1 and 3; see Figure 4B). Each IPSP decayed to almost zero before another IPSP was generated post-synaptically on PC dendrites.
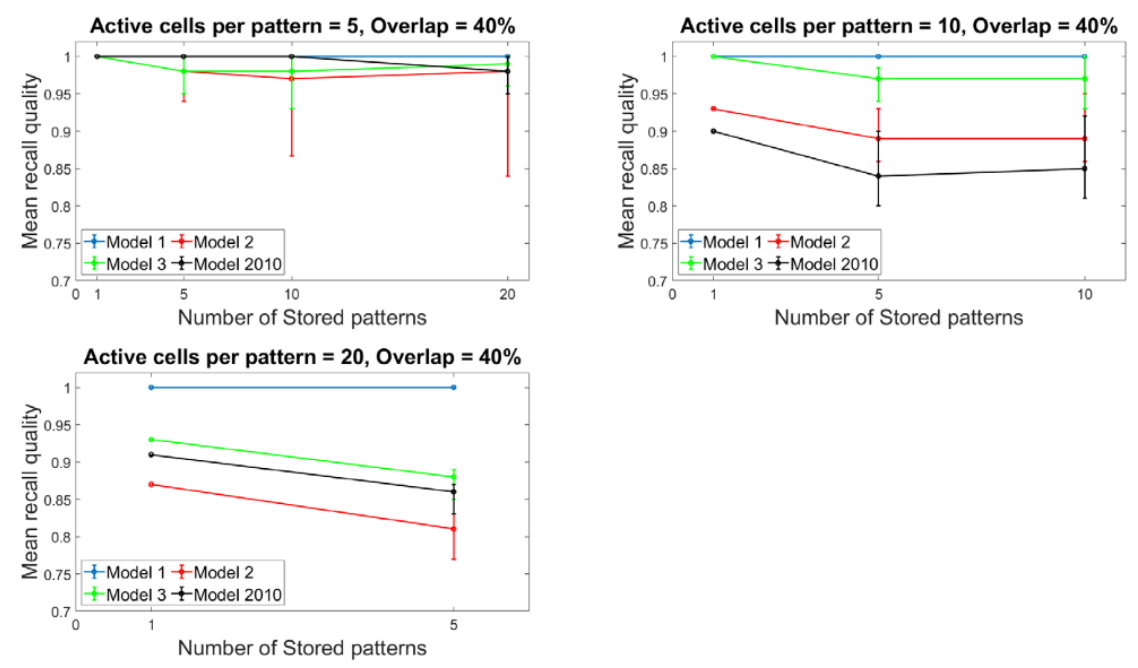
Fig. 6. Mean recall quality of 'model 1', 'model 2', 'model 3' and 'model 2010' as a function of number of stored patterns. Variable number of 'active cells per pattern' $(5,10,20)$ in a network of 100 PCs with $40 \%$ overlap.

\section{Conclusions}

A bio-inspired neural model of hippocampal CA1 region [1] was employed to systematically evaluate its mean recall quality against number of stored patterns, percent overlaps and 'active cells per pattern'. Modulation of selective excitatory and inhibitory pathways to BSC and PC dendrites as more and more patterns were stored in the network of $100 \mathrm{CA} 1-\mathrm{PCs}$ resulted into three models, the performances of which were compared to that of the original 2010 model of Cutsuridis et al. [1]. Of the three models tested, 'Model's 1' performance was excellent across all conditions, whereas 'Model's 2' performance was consistently the worst. A key finding of ours is that the number of 'active cells per pattern' has a massive effect on the recall quality of the network regardless of how many patterns are stored in it. As the number of dedicated cells representing a memory ('active cells per pattern') decrease, the memory capacity of the CA1PC network increases, so interference effects between stored patterns decrease, and mean recall quality increases. Another key finding of ours is that increased firing frequency response of a presynaptic inhibitory cell (BSC) inhibiting a network of PCs has a better success at removing spurious activity at the network level and thus improving recall quality than an increased synaptic efficacy of a presynaptic inhibitory cell (BSC) on a postsynaptic $\mathrm{PC}$ while keeping its presynaptic firing rate fixed.

\section{References}

1. Cutsuridis, V., Cobb, S., Graham, B.P.: Encoding and retrieval in a model of the hippocampal CA1 microcircuit. Hippocampus 20, 423-446 (2010).

2. Megias, M., Emri, Z.S., Freund, T.F., Gulyas, A.I.: Total number and distribution of inhibitory and excitatory synapses on hippocampal CA1 pyramidal cells. Neuroscience 102(3), 527-540 (2001)

3. Gulyas, A.I., Megias, M. Emri, Z., Freund, T.F.: Total number and ratio of excitatory and inhibitory synapses converging onto single interneurons of different types in the CA1 areas of the rat hippocampus. J. Neurosci. 19(22), 10082-10097 (1999).

4. Poirazi, P., Brannon, T., Mel, B.W.: Arithmetic of subthreshold synaptic summation in a model of CA1 pyramidal cell. Neuron 37, 977-987 (2003a)

5. Poirazi, P., Brannon, T., Mel, B.W.: Pyramidal neuron as a 2-layer neural network. Neuron 37, 989-999 (2003b)

6. Santhakumar, V., Aradi, I., Soltetz, I.: Role of mossy fiber sprouting and mossy cell loss in hyperexcitability: a network model of the dentate gyrus incorporating cell types and axonal topography. J. Neurophysiology 93, 437-453 (2005)

7. Buhl, E.H., Szilágyi, T., Halasy, K., Somogyi, P.: Physiological properties of anatomically identified basket and bistratified cells in the CA1 area of the rat hippocampus in vitro. Hippocampus 6(3), 294-305 (1996). 
8. Hines, M.L., Carnevale, T.: The NEURON simulation environment. Neural Comput. 9, 1179-1209 (1997).

9. Amaral, D., Lavenex, P.: Hippocampal neuroanatomy. In: Andersen, P., Morris, R., Amaral, D., Bliss, T., O'Keefe, J. (eds.) The Hippocampus Book, pp. 37-114. Oxford University press, Oxford (2007).

10. Andersen, P., Morris, R., Amaral, D., Bliss, T., O'Keefe, J.: The hippocampus book. Oxford University press, Oxford (2007)

11. Buhl, E.H., Halasy, K., Somogyi, P.: Diverse sources of hippocampal unitary inhibitory postsynaptic potentials and the number of synaptic release sites. Nature 368, 823-828 (1994)

12. Cutsuridis, V.: Improving the recall performance of a brain mimetic microcircuit model. Cogn Comput. 11, 644-655 (2019)

13. Buhl, E.H., Han, Z.S., Lorinczi, Z., Stezhka, V.V., Kapnup, S.V., Somogyi, P.: Physiological properties of anatomically identified axo-axonic cells in the rat hippocampus. J. Neurophys. 71(4), 1289-1307 (1994)

14. Pospischil, M., Toledo-Rodriguez, M., Monier, C., Piwkowska, Z., Bal, T., Frégnac, Y., Markram, H., Destexhe, A.: Minimal Hodgkin-Huxley type models for different classes of cortical and thalamic neurons. Biol Cybern. 99(4-5), 427-41 (2008).

15. Saraga, F., Wu, C.P., Zhang, L., Skinner, F.K.: Active dendrites and spike propagation in multicompartmental models of oriens-lacunosum/moleculare hippocampal interneurons. J Physiol 552, 673-689 (2008).

16. Borhegyi, Z., Varga, V., Szilagyi, N., Fabo, D., Freund, T.F.: Phase segregation of medial septal GABAergic neurons during hippocampal theta activity. J Neurosci 24, 8470-8479 (2004)

17. Petersen, C.C.H., Malenka, R.C., Nicoll, R.A., Hopfield, J.J.: All-or none potentiation at CA3-CA1 synapses. Proc Natl Acad Sci USA 95, 4732-4737 (1998)

18. Suzuki, W.A.: Making new memories: The role of the hippocampus in new associative learning. Annals of the New York Academy of Sciences 1097, 1-11 (2007).

19. Steinbuch, K.: Non-digital learning matrices as preceptors. Kybernetik 1, 117-124 (1961).

20. Willshaw, D., Buneman, O., Longuet-Higgins, H.: Non-holographic associative memory. Nature 222, 960-962 (1969).

21. Klausberger, T., Magill, P.J., Marton, L.F., David, J., Roberts, B., Cobden, P.M., Buzsaki, G., Somogyi, P.: Brain-state- and cell-type-specific firing of hippocampal interneurons in vivo. Nature 421, 844-848 (2003).

22. Klausberger, T., Marton, L.F., Baude, A., Roberts, J.D., Magill, P.J., Somogyi, P.: Spike timing of dendrite-targeting bistratified cells during hippocampal network oscillations in vivo. Nat Neurosci 7, 41-47 (2004) 